\title{
VARIATIONAL MOTION DESIGN
}

\author{
Helmut Pottmann and Michael Hofer \\ Geometric Modeling and Industrial Geometry Group \\ Vienna University of Technology, A-1040 Wien, Austria \\ pottmann, hofer@geometrie.tuwien.ac.at \\ Bahram Ravani \\ Department of Mechanical and Aeronautical Engineering \\ University of California, Davis, CA 95616, USA \\ bravani@ucdavis.edu
}

\begin{abstract}
We discuss the following motion design problem: Given are $N$ positions $\Sigma\left(t_{i}\right)$ of a moving body $\Sigma^{0} \subset \mathbb{R}^{3}$ at time instances $t_{i}$. Compute a smooth rigid body motion $\Sigma(t)$ which interpolates or approximates the given key or control positions $\Sigma\left(t_{i}\right)$. Moreover, the motion shall minimize a certain energy which is expressed with help of the energies of trajectories of points on the moving body. Based on very recent results on splines in manifolds we present a characterization of motions which are analogous to known energy minimizing spline curves, such as $C^{2}$ cubic splines or splines in tension.
\end{abstract}

Keywords: Motion design, motion planning, spline motion, splines in manifolds, variational design, screw theory

\section{Introduction}

Smooth interpolation or approximation of given positions of a moving body is a fundamental task in computer animation and robot motion planning (Latombe, 2001). There exists a large body of literature dealing with this subject, which is rooted in kinematics and Computer Aided Geometric Design (for a recent survey, see Jüttler and Wagner, 2002).

From a more abstract point of view, the problem is a curve interpolation or approximation problem in a manifold. In the present case, this manifold is formed by the elements of the group SE(3) of Euclidean congruence transformations. Depending on the application, various embeddings in a higher dimensional space are appropriate.

Recent work (Belta and Kumar, 2002; Hofer et al., 2004; Wallner, 2004) uses an embedding as 6-dimensional manifold $M^{6}$ in a 12-dimensional affine space, which arises as kinematic image space of affine maps in $\mathbb{R}^{3}$. With an appropriate Euclidean metric in $\mathbb{R}^{12}$, the orthogonal 
projection onto $M^{6}$ can be computed explicitly. Now smooth motions arise via the projection of smooth curves $\mathbf{c}(t) \subset \mathbb{R}^{12}$ onto $M^{6}$. If $\mathbf{c}(t)$ minimizes an energy functional, its projection $\overline{\mathbf{c}} \subset M^{6}$ is in general not a minimizer. Therefore, this simple projection principle is not suitable for variational motion design. For contributions to the latter topic, see Barr et al., 1992; Jüttler and Wagner, 2002; Park and Ravani, 1997; Ramamoorthi and Barr, 1997.

In continuation of work started in Hofer et al., 2004, we discuss here variational motion design as a variational curve design problem on $M^{6} \subset \mathbb{R}^{12}$. Very recent general results on energy minimizing splines in manifolds (Pottmann and Hofer, 2004) are used to characterize energy minimizing motions. The energy is formulated with help of the energies of trajectories of a finite or infinite set of points (feature points) on the moving body. We find characterizations which are analogous to elegant known results in classical spline theory. For example, the $L^{2}$ norm of the second derivative as an energy leads in the unrestricted classical curve interpolation case to cubic $C^{2}$ splines; for motion interpolation, we obtain $C^{2}$ motions for which - at each time instant - the force system, defined by the 4 -th derivative vectors attached to the feature points, is in balance. Geodesic motions in the present setting appear as free motions in the sense of mechanics (Arnol'd, 1989).

The focus of the present paper lies on the description of the theoretical framework and the derivation of the main characterization of energy minimizing motions. We address the computation, which is a special case of the computation of splines in manifolds, only briefly, and finally present illustrative examples.

\section{The Group of Euclidean Motions Embedded in the Affine Group}

Consider a rigid body moving in Euclidean three-space $E^{3}$. We use Cartesian coordinates and denote points of the moving system $\Sigma^{0}$ by $\mathbf{x}^{0}, \mathbf{y}^{0}, \ldots$, and points of the fixed system by $\mathbf{x}, \mathbf{y}$, and so on.

A one-parameter motion $\Sigma^{0} / \Sigma$ is a smooth family of Euclidean congruence transformations depending on a parameter $t$ which can be thought of as time. A point $\mathbf{x}^{0}$ of $\Sigma^{0}$ is, at time $t$, mapped to the point

$$
\mathbf{x}(t)=A(t) \cdot \mathbf{x}^{0}+\mathbf{a}_{0}(t)
$$

of $\Sigma$, where $A(t) \in S O(3)$ and $\mathbf{a}_{0}(t) \in \mathbb{R}^{3}$.

If we do not impose any restriction to the matrix $A$ in Eq. (1), we get, for each $t$, an affine map. 
In the following, we will use a kinematic mapping that views affine maps as points in 12-dimensional affine space. For that, consider the affine map $\mathbf{x}=\alpha\left(\mathbf{x}^{0}\right)=\mathbf{a}_{0}+A \cdot \mathbf{x}^{0}$. Let us denote the three column vectors of $A$ as $\mathbf{a}_{1}, \mathbf{a}_{2}, \mathbf{a}_{3}$. They describe the images of the basis vectors of $\Sigma^{0}$ in $\Sigma$. Of course, we have $\mathbf{x}=\mathbf{a}_{0}+x_{1}^{0} \mathbf{a}_{1}+x_{2}^{0} \mathbf{a}_{2}+x_{3}^{0} \mathbf{a}_{3}$. Now we associate with the affine map $\alpha$ a point in 12-dimensional affine space $\mathbb{R}^{12}$, represented by the vector $\mathrm{A}=\left(\mathbf{a}_{0}, \ldots, \mathbf{a}_{3}\right)$.

The images of Euclidean congruence transformations (rigid body motions) $\alpha \in S E(3)$ form a 6-dimensional manifold $M^{6} \subset \mathbb{R}^{12}$. Its 6 equations are given by the orthogonality conditions of $A$, i.e., $\mathbf{a}_{i} \cdot \mathbf{a}_{j}=$ $\delta_{i j}, i=1,2,3$.

It will be necessary to introduce a meaningful metric in $\mathbb{R}^{12}$. Following Hofer et al., 2004, this is done with help of a collection $X$ of points $\mathbf{x}_{1}^{0}, \mathbf{x}_{2}^{0}, \ldots, \mathbf{x}_{N}^{0}$ in the moving system (body), which shall be called feature points henceforth. The squared distance between two affine maps $\alpha$ and $\beta$ is now defined as sum of squared distances of feature point positions after application of $\alpha$ and $\beta$, respectively,

$$
d^{2}(\alpha, \beta)=\|\mathrm{A}-\mathrm{B}\|^{2}:=\sum_{i}\left[\alpha\left(\mathbf{x}_{i}^{0}\right)-\beta\left(\mathbf{x}_{i}^{0}\right)\right]^{2} .
$$

With $\mathrm{A}=\left(\mathbf{a}_{0}, \ldots, \mathbf{a}_{3}\right), \mathrm{B}=\left(\mathbf{b}_{0}, \ldots, \mathbf{b}_{3}\right), \mathrm{C}:=\mathrm{A}-\mathrm{B}=\left(\mathbf{c}_{0}, \ldots, \mathbf{c}_{3}\right)$, and $\mathbf{x}_{i}^{0}=\left(x_{i, 1}^{0}, x_{i, 2}^{0}, x_{i, 3}^{0}\right)$ the distance becomes

$$
\|\mathrm{A}-\mathrm{B}\|^{2}=\|\mathrm{C}\|^{2}=\sum_{i}\left[\mathbf{c}_{0}+x_{i, 1}^{0} \mathbf{c}_{1}+x_{i, 2}^{0} \mathbf{c}_{2}+x_{i, 3}^{0} \mathbf{c}_{3}\right]^{2}=\mathrm{C}^{T} \cdot M \cdot \mathrm{C}
$$

This expression with help of a positive definite symmetric matrix $M$ immediately reveals the following result (Hofer et al., 2004): The metric (2) in the space of affine maps only depends on the barycenter $\mathbf{s}_{x}=$ $(1 / N) \sum_{i} \mathbf{x}_{i}^{0}$ and on the inertia tensor

$$
J:=\sum_{i} \mathbf{x}_{i}^{0} \cdot \mathbf{x}_{i}^{0^{T}}
$$

of the set of feature points $\mathbf{x}_{i}^{0}$ in the moving system. $\mathbb{R}^{12}$ equipped with this metric is a Euclidean space $E^{12}$.

We also see that we need not use unit point masses at a discrete number of feature points. We could instead work with another positive measure on a domain of interest $D$ (the moving body) in $\Sigma^{0}$, e.g. the Lebesgue measure times the characteristic function $\chi_{d}$ of $D$. Of course, we then replace summation in (2) by integration. 
By a well-known result from mechanics, we can replace the points $\mathbf{x}_{1}^{0}, \ldots, \mathbf{x}_{N}^{0}$ by the six special points

$$
\mathbf{s}_{x} \pm \sqrt{\frac{\lambda_{j}}{2}} \mathbf{e}^{j}, \quad j=1,2,3,
$$

without changing the barycenter and the inertia tensor of $X$. There, $\lambda_{1}, \lambda_{2}, \lambda_{3}$ and $\mathbf{e}^{1}, \mathbf{e}^{2}, \mathbf{e}^{3}$ are the eigenvalues and corresponding unit eigenvectors of the matrix $J$. Let us choose the barycenter as origin in the moving system and the eigenvectors of $J$ as coordinate axes. Then the six points have coordinates $\left( \pm f_{1}, 0,0\right),\left(0, \pm f_{2}, 0\right),\left(0,0, \pm f_{3}\right)$, and the norm in $\mathbb{R}^{12}$ becomes

$$
\|\mathrm{C}\|^{2}=6 \mathbf{c}_{0}^{2}+2 \sum_{i=1}^{3} f_{i}^{2} \mathbf{c}_{i}^{2}
$$

For the following considerations it will be important to know about the tangent spaces at points $\mathrm{A} \subset M^{6}$ and to characterize orthogonality to these tangent spaces.

A tangent vector at an arbitrary point $A \in E^{12}$ can be interpreted in $E^{3}$ as a velocity vector field of an affine motion. In particular, a tangent vector of $M^{6}$ belongs to a velocity vector field of a Euclidean motion, which is of the form $\mathbf{v}(\mathbf{x})=\overline{\mathbf{c}}+\mathbf{c} \times \mathbf{x}$. The coordinate representation of this tangent vector in $E^{12}$, attached to $\mathrm{A}=\left(\mathbf{a}_{0}, \ldots, \mathbf{a}_{3}\right) \in M^{6}$, reads

$$
\mathrm{T}=\left(\overline{\mathbf{c}}+\mathbf{c} \times \mathbf{a}_{0}, \mathbf{c} \times \mathbf{a}_{1}, \ldots, \mathbf{c} \times \mathbf{a}_{3}\right) .
$$

We would like to express orthogonality of an arbitrary vector $\mathrm{D}=$ $\left(\mathbf{d}_{0}, \mathbf{d}_{1}, \ldots, \mathbf{d}_{3}\right) \in \mathbb{R}^{12}$ to the tangent space at $A$. If we align origin and axes of the coordinate system in $\Sigma^{0}$ with center and axes of the inertia ellipsoid, the inner product is expressed in view of (6) as

$$
\langle\mathrm{D}, \mathrm{T}\rangle=6 \mathbf{d}_{0} \cdot\left(\overline{\mathbf{c}}+\mathbf{c} \times \mathbf{a}_{0}\right)+2 \sum_{i=1}^{3} f_{i}^{2} \mathbf{d}_{i} \cdot\left(\mathbf{c} \times \mathbf{a}_{i}\right) .
$$

This equation may also be written as

$$
\langle\mathrm{D}, \mathrm{T}\rangle=6 \mathbf{d}_{0} \cdot \overline{\mathbf{c}}+6 \mathbf{c} \cdot\left(\mathbf{a}_{0} \times \mathbf{d}_{0}\right)+2 \mathbf{c} \sum_{i=1}^{3} f_{i}^{2}\left(\mathbf{a}_{i} \times \mathbf{d}_{i}\right) .
$$

Let us attach to the six feature point positions $\mathbf{a}_{0} \pm f_{i} \mathbf{a}_{i}, i=1,2,3$, the vectors of the linear vector field determined by $D \in \mathbb{R}^{12}$. These vectors are $\mathbf{d}_{0} \pm f_{i} \mathbf{d}_{i}$, and shall be interpreted as forces (for such concepts 
from statics, see e.g. Pottmann and Wallner, 2001, pp. 191-194). The moments of these forces are

$$
\left(\mathbf{a}_{0} \pm f_{i} \mathbf{a}_{i}\right) \times\left(\mathbf{d}_{0} \pm f_{i} \mathbf{d}_{i}\right) .
$$

The sums of force vectors and moment vectors is the screw

$$
\left(\mathbf{s}_{d}, \overline{\mathbf{s}}_{d}\right)=\left(6 \mathbf{d}_{0}, 6 \mathbf{a}_{0} \times \mathbf{d}_{0}+2 \sum_{i=1}^{3} f_{i}^{2}\left(\mathbf{a}_{i} \times \mathbf{d}_{i}\right)\right) .
$$

The screw resulting from the action of an instantaneous affine motion (linear vector field) on these points is the same whether we use the 6 special feature points or the original set of feature points (or the mass distribution). We call this the screw or force system $\mathrm{S}_{d}$ induced by the linear vector field $\mathrm{D}$.

The inner product (8) between $\mathrm{T}$ and $\mathrm{D}$ is now expressed as

$$
\langle\mathrm{D}, \mathrm{T}\rangle=\overline{\mathbf{c}} \cdot \mathbf{s}_{d}+\mathbf{c} \cdot \overline{\mathbf{s}}_{d} .
$$

This is the virtual work done by the force system $\mathrm{S}_{d}$ on the body which moves instantaneously with the velocity field determined by T. Orthogonality between $\mathrm{D}$ and all $\mathrm{T}$ requires $\left(\mathbf{s}_{d}, \overline{\mathbf{s}}_{d}\right)=0$, i.e., balance of the induced force system $S_{d}$. Thus, we have proved the following result.

Theorem $1 A$ vector $\mathrm{D} \in \mathbb{R}^{12}$, attached to a point $\mathrm{A} \in M^{6}$, is orthogonal to $M^{6} \subset E^{12}$ iff the force system $\mathrm{S}_{d}$ induced by $\mathrm{D}$ is in balance.

\section{Variational Motion Design as Variational Curve Design on $M^{6} \subset E^{12}$}

A Euclidean one-parameter motion corresponds to a curve on the manifold $M^{6} \subset E^{12}$. The design of such curves via projection onto $M^{6}$ is a principle of transfer from curve design to motion design (Hofer et al., 2004). Such an approach, however, does not preserve minimum energy properties. Thus, we are now dealing with the problem of constructing energy minimizing interpolating spline curves on $M^{6}$. In this way, we are computing energy minimizing Euclidean motions which interpolate given positions.

Consider an $m$-dimensional manifold $\Phi$, embedded in Euclidean $\mathbb{R}^{n}$, $m<n$. Moreover, a sequence of points $\mathbf{p}_{i} \in \Phi, i=1, \ldots, N$ and real numbers $t_{1}<\cdots<t_{N}$ are given. We are seeking interpolating splines in the manifold.

Let us recall the situation, where we are not confined to a manifold: Among all curves $\mathbf{x}(t) \subset \mathbb{R}^{n}$, whose first and second derivative satisfy 
$\dot{\mathbf{x}} \in A C(I), \ddot{\mathbf{x}} \in L^{2}(I)$ on $I=\left[t_{1}, t_{N}\right]$, and which interpolate the given data, $\mathbf{x}\left(t_{i}\right)=\mathbf{p}_{i}$, the unique minimizer of

$$
E_{2}(\mathbf{x})=\int_{t_{1}}^{t_{N}} \ddot{\mathbf{x}}^{2} d t
$$

is the interpolating $C^{2}$ cubic spline $\mathbf{c}(t)$.

Pottmann and Hofer, 2004 extended this well-known result to the case where the admissible curves $\mathbf{x}(u)$ are restricted to the given manifold $\Phi$. We are considering the restriction to $\Phi$ as a constraint, rather than formulating the problem in terms of the intrinsic geometry of the manifold. The solution can be characterized as follows (Pottmann and Hofer, 2004):

Theorem 2 Consider real numbers $t_{1}<\ldots<t_{N}$ and points $\mathbf{p}_{1}, \ldots, \mathbf{p}_{N}$ on an m-dimensional $C^{4}$ manifold $\Phi$ in Euclidean $\mathbb{R}^{n}$. Then among all $C^{1}$ curves $\mathbf{x}:\left[t_{1}, t_{N}\right] \rightarrow \Phi \subset \mathbb{R}^{n}$, whose restrictions to the intervals $\left[t_{i}, t_{i+1}\right], i=1, \ldots, N-1$ are $C^{4}$ and which interpolate the given points, $\mathbf{x}\left(t_{i}\right)=\mathbf{p}_{i}, i=1, \ldots, N$, a curve $\mathbf{c}$ which minimizes the functional $E_{2}$ of Eq. (11) is $C^{2}$ and possesses segments $\mathbf{c} \mid\left[t_{i}, t_{i+1}\right]$, whose fourth derivative vectors are orthogonal to $\Phi$. Moreover, at the end points $\mathbf{p}_{1}=\mathbf{c}\left(t_{1}\right)$ and $\mathbf{p}_{N}=\mathbf{c}\left(t_{N}\right)$ of the solution curve, the second derivative vector is orthogonal to $\Phi$.

We are viewing $t_{i}$ as given time instances, at which given positions, i.e., points $\mathrm{P}_{i} \in M^{6}$, have to be interpolated. The energy (11) of a curve $\mathrm{X}(t) \subset E^{12}$ uses the norm induced by (2) and thus it may also be interpreted as sum of the corresponding energies $\left(L^{2}\right.$ norms of the second derivatives) of the feature point trajectories.

According to Theorem 2, the solution curve $\mathrm{C}(t) \subset M^{6}$ has 4-th derivative vectors $\mathrm{C}^{(4)}(t)$, which are orthogonal to $M^{6}$. The force system $\mathrm{S}_{4}(t)$ induced by $\mathrm{C}^{(4)}(t)$ consists of the fourth derivative vectors of the feature point trajectories at a given instant $t$. Using Theorems 1 and 2, we obtain the following result.

Theorem 3 Consider $N$ input positions, corresponding time instances $t_{i}$ and differentiability assumptions as in Theorem 2. Then, an interpolating motion minimizing the sum of energies ( $L^{2}$ norms of the second derivatives) of the feature point trajectories is characterized as follows. The motion is $C^{2}$, has at each time instant $t \neq t_{i}$ a balanced 4 -th derivative force system $\mathrm{S}_{4}(t)$, and at the end positions balanced force systems $\mathrm{S}_{2}\left(t_{1}\right), \mathrm{S}_{2}\left(t_{N}\right)$ of second derivatives. In particular, the trajectory of the barycenter of the feature points is an interpolating cubic $C^{2}$ spline. 
The result on the trajectory of the barycenter follows from the vanishing of the force components $\mathbf{s}_{k}$ of the involved $k$-th derivative systems $\mathrm{S}_{k}=\left(\mathbf{s}_{k}, \overline{\mathbf{s}}_{k}\right)$.

We see that these motions somehow balance the deviations of the point trajectories from $C^{2}$ cubic splines; there the 4-th derivatives would vanish everywhere. Note that a motion with only $C^{2}$ cubic spline trajectories must be translational (the image curve lies in a 3-dimensional affine subspace contained in $M^{6}$ and is a cubic spline itself).

It is also quite natural that the moving body via its mass distribution (barycenter and inertia tensor) enters the variational formulation and the interpretation of the solution. That the present approach is natural from the viewpoint of mechanics and kinematics, is also nicely seen if we replace the energy in (11) by the $L^{2}$ norm of the first derivative,

$$
E_{1}(\mathbf{x})=\int_{t_{1}}^{t_{N}} \dot{\mathbf{x}}^{2} d t
$$

Moreover, we just prescribe the two end positions. Then, one finds with a known counterpart of Theorem 2 (see e.g. Pottmann and Hofer, 2004) a curve $\mathrm{C}(t) \subset M^{6}$, whose second derivative vectors $\ddot{C}$ are orthogonal to $M^{6}$. From this we conclude immediately that the minimizers of $E_{1}$ are geodesics on $M^{6}$ in a scaled arc length parameterization, i.e., $\|\dot{\mathrm{C}}\|=$ const. This proves the following theorem.

Theorem 4 Motions which join two given positions and arise from minimization of (12) correspond to geodesics on $M^{6}$, parameterized by a constant multiple of arc length. At any time instant, such a geodesic motion possesses a balanced force system $\mathrm{S}_{2}(t)$ of second derivatives. The trajectory of the barycenter of the feature point set on the moving body is a straight line traced with constant speed. These motions are free motions of a body in the sense of mechanics.

Note that a helical motion is a geodesic motion between two positions $\Sigma\left(t_{1}\right)$ and $\Sigma\left(t_{2}\right)$, if the positions of the inertia ellipsoids share a common axis, and if this is the axis of the helical motion joining the two positions. For more results on free motions, we refer to Arnol'd, 1989.

By minimization of a combination $E_{t}:=E_{2}+w E_{1}$ with a constant positive factor $w$ one obtains the counterparts of splines in tension for motions. These are characterized as in Theorem 3, but instead of a balanced 4-th derivative force system, the linearly combined force system $\mathrm{S}_{4}-w \mathrm{~S}_{2}$ is in balance. A proof follows from results in (Pottmann and Hofer, 2004). Various other spline types, interpolating or approximating, can be transferred to motion design within the present setting. This will be developed in the future. 
Note that the functionals we are considering are also optimizing the parameterization. This is useful for motion design where the time $t$ as parameter plays an important role for applications.

Since even for geodesics we do not have simple results on uniqueness, the more involved case of splines will hardly allow us a characterization of situations with a unique solution. For a proof of the existence of the solution, we refer to (Bohl, 1999; Wallner, 2003).

\section{Computational Approach and Examples}

Although the presented spline motions possess nice geometric characterizations, the problem is still nonlinear and does not admit an explicit solution as in the unrestricted curve design case. Thus, we have to use numerical algorithms based on a discretization. Geometrically this means that we replace the curve $\mathrm{C}(t) \subset M^{6}$ by a sufficiently dense polygon $P_{c}$ with vertices $C_{i} \in M^{6}$. These vertices represent positions of the motion at discrete time instances.

Discretizing a quadratic functional, such as $E_{1}, E_{2}, E_{t}$, results in a quadratic function in the coordinates of the vertices $C_{i}$ of the approximating polygon $P$. Since the $C_{i}$ 's are restricted to $M^{6}$, we end up with the constrained minimization of a quadratic function in a rather high dimensional space. From a geometric viewpoint, we have to construct the closest point $\mathbf{p}^{*}$ on some manifold $S$ to a given point $\mathbf{p}$. Here, $\mathbf{p}$ represents the unrestricted (discretized) spline curve in $E^{12}$. For the solution of this problem, we developed a geometrically motivated optimization algorithm of the quasi-Newton type (Hofer and Pottmann, 2004). It has been used for the computation of the examples presented in Figs. 1,2.

Another computational approach directly uses the characterization of the motions in a discretized way. For example, a motion minimizing $E^{2}$ has a balanced 4th central difference screw at any instance in the time discretization. This results in a system of nonlinear equations, which can be solved with a Newton iteration. In view of the bad global behaviour of a pure Newton algorithm, we used the quasi-Newton approach mentioned above.

\section{Conclusions and Future Research}

We have used a kinematic mapping of $\mathrm{SE}(3)$ to points of a six dimensional manifold $M^{6}$ embedded in 12-dimensional Euclidean space $E^{12}$. The latter space corresponds to affine maps, and the metric therein is defined naturally with help of feature points (a mass distribution) of the moving body. Variational motion design is thus transferred to variational curve design on $M^{6}$. Based on recent results on splines in manifolds, 


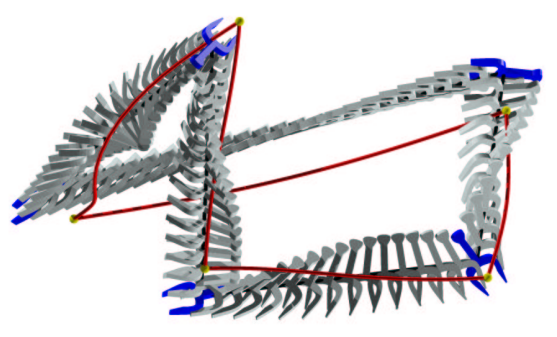

(a)

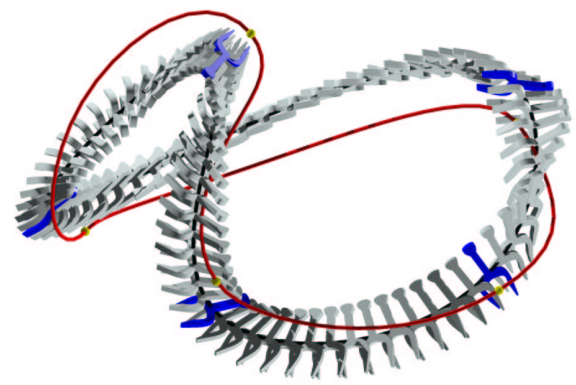

(c)

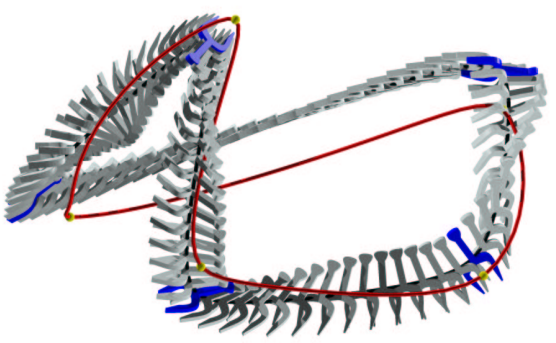

(b)

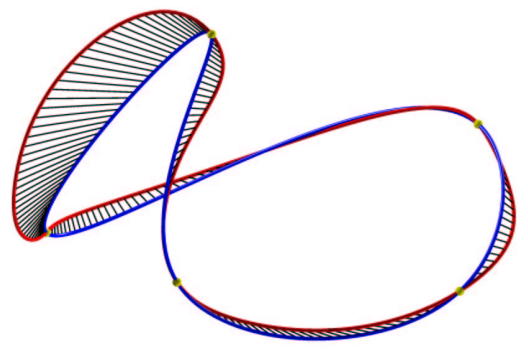

(d)

Figure 1. Cyclic motion of a robot gripper interpolating 5 positions (dark), and the paths of the barycenter and the feature point $\left(f_{1}, 0,0\right)$ : Motion minimizing (a) $E_{1}$, (b) $E_{t}$ with $w=0.05$, (c) $E_{2}$; (d) Path of feature point compared to cubic spline.

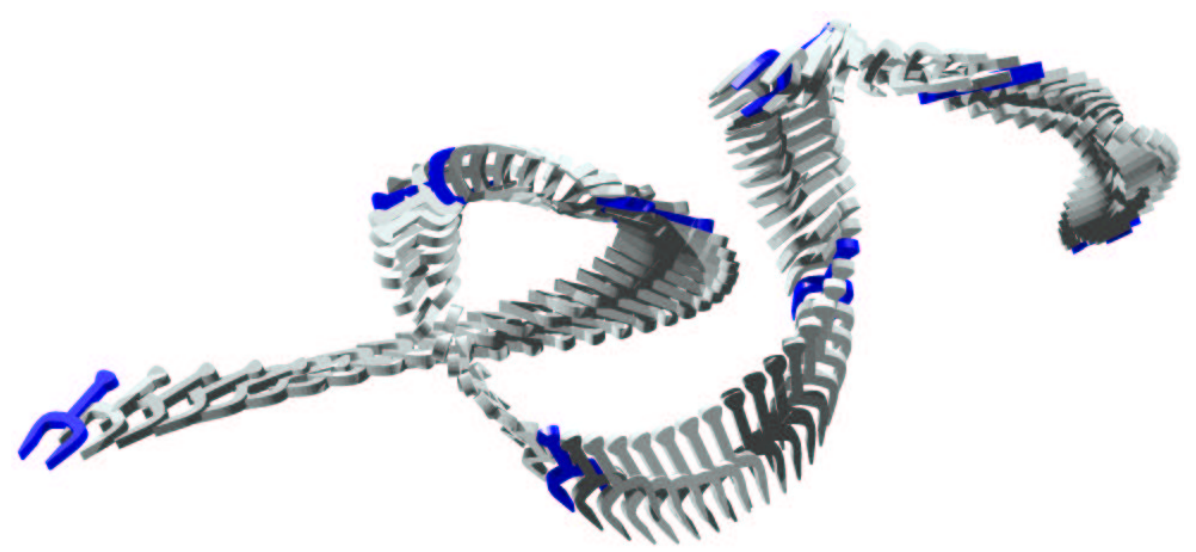

Figure 2. Open motion interpolating 8 positions and minimizing $E_{2}$. 
we could characterize and compute the solutions of several variational motion design problems that generalize known variational curve design schemes. The present contribution is not aiming at a special representation of the solution, e.g. in NURBS form, which could be obtained after the design by an approximation scheme (see Jüttler and Wagner, 2002). Future research will be devoted to a more efficient computation based on a quadratically convergent Newton-type iteration, to motion smoothing, and to obstacle avoidance in motion design.

\section{Acknowledgements}

This research has been supported by the Austrian Science Fund (FWF) under grant P16002-N05, and in part by California Department of Transportation through University of California-Davis.

\section{References}

Arnol'd, V.I. (1989), Mathematical methods of classical mechanics. 2nd ed., Springer, New York.

Barr, A.H., Currin, B., Gabriel, S., and Hughes, J.F. (1992), Smooth interpolation of orientations with angular velocity constraints using quaternions, Computer Graphics 26 (SIGGRAPH '92), pp. 313-320.

Belta, C., and Kumar, V. (2002), An SVD-projection method for interpolation on SE(3), IEEE Trans. on Robotics and Automation 18, 334-345.

Bohl, H. (1999), Kurven minimaler Energie auf getrimmten Flächen, PhD thesis, Stuttgart.

Hofer, M., and Pottmann, H. (2004), Energy-minimizing splines in manifolds, submitted for publication.

Hofer, M., Pottmann, H., and Ravani, B. (2004), From curve design algorithms to the design of rigid body motions, The Visual Computer, to appear.

Jüttler, B., and Wagner, M. (2002), Kinematics and Animation, In: G. Farin, J. Hoschek, M.S. Kim, eds., Handbook of Computer Aided Geometric Design, Elsevier, pp. 723748.

Latombe, J.-C. (2001), Robot Motion Planning, 6th printing, Kluwer.

Park, F.C., and Ravani, B. (1997), Smooth invariant interpolation of rotations, ACM Transactions on Graphics 16, pp. 277-295.

Pottmann, H., and Hofer, M., (2004), A variational approach to spline curves on surfaces, submitted for publication.

Pottmann, H., and Wallner, J. (2001), Computational Line Geometry, Springer-Verlag, Berlin/Heidelberg/New York.

Ramamoorthi, R., and Barr, A., (1997), Fast construction of accurate quaternion splines, Computer Graphics (Proceedings of SIGGRAPH 1997), pp. 287-292.

Wallner, J. (2003), Energy minimizing curves on surfaces, Preprint, Institute of Geometry, Vienna Univ. of Technology, December 2003.

Wallner, J. (2004), Gliding spline motions and applications, Computer Aided Geom. Design, vol. 21, pp. 3-21. 